\title{
The Effects of Silicone Oil Endotamponade on Subfoveal Choroidal Thickness after Pars Plana Vitrectomy for Rhegmatogenous Retinal Detachment
}

\author{
Selim Bolukbasi, () Burak Erden, (1) Akin Cakir
}

Department of Ophthalmology, University of Health Sciences Okmeydani Training and Research Hospital, Istanbul, Turkey

\begin{abstract}
Objectives: To evaluate the potential effects of 1000 -cSt silicone oil (SO), which is used for pars plana vitrectomy (PPV) surgery on subfoveal choroidal thickness.

Methods: In this retrospective study, 20 eyes of 20 patients who had undergone PPV and SO injection surgery for maculaoff rhegmatogenous retinal detachment were included. Complete ophthalmologic examinations were performed before the surgery, one month, three months after PPV and two months after silicone oil removal. After one month following PPV and SO injection and two months following silicone oil removal, subfoveal choroidal thickness was evaluated using EDI (enhanced depth imaging) mode of spectral-domain optical coherence tomography.

Results: Subfoveal choroidal thickness measurements decreased statistically significantly at two months after silicone oil removal compared to the first month of PPV and SO injection. The mean subfoveal choroidal thickness was $284.2 \pm 54.2$ $\mu \mathrm{m}$ at one month after PPV and SO injection, while it was $258.8 \pm 52.8 \mu \mathrm{m}$ at two months after SO removal $(\mathrm{P}<0.00 \mathrm{l})$.

Conclusion: After the removal of SO subfoveal choroidal thickness measurements decreased, the changes of subfoveal choroidal thickness measurements may be a parameter for the timing of SO removal.
\end{abstract}

Keywords: pars plana vitrectomy, rhegmatogenous retinal detachment, subfoveal choroidal thickness, silicone oil.

\section{Introduction}

In the surgical treatment of rhegmatogenous retinal detachment (RRD), silicone oil (SO) injection following pars plana vitrectomy (PPV) has been preferred by many surgeons for years. In the relevant literature, there are many studies regarding the side effects of SO on the cornea, optic nerve, trabecular meshwork, retina and lens $(\mathrm{I}-4)$. Especially the usage of optical coherence tomography (OCT) is more common, and the potential effects of SO on the retina can be monitored more detailed. There are some reports regarding vision loss related to SO. Progressive thinning of the inner retinal layers in the macular region has been shown with
OCT (5). Also, there are some studies showing SO related optic nerve degeneration (6). However, we should note that there is not still a consensus on the timing of SO removal. In the past, timing of SO removal was more preferred at six months after PPV, but the complication incidence may increase with the time of SO removal delayed. Nowadays, many surgeons recommend removal of $\mathrm{SO}$ at four months or even at two months after surgery to be aware of the SO side effects.

The choroid supplies the outer retinal layers, including outer plexiform, outer nuclear, photoreceptor and retinal pigment epithelium (RPE) with oxygen and nutrients. Choroid also plays a role in the mechanisms of many dis- 
eases, and it can be evaluated more detailed with EDI (enhanced depth imaging) mode of OCT.

The present study aims to investigate the potential effects of SO duration on subfoveal choroidal thickness in patients with macula-off RRD to determine the proper time for SO removal.

\section{Methods}

In this retrospective, observational study, medical records of patients having macula-off RRD, who were treated with PPV and a 1000-cSt SO endotamponade followed by subsequent SO removal between 06.2016-10.2018 were reviewed. The first and third month following PPV with SO injection and second month following SO removal, best-corrected visual acuity (BCVA) with Snellen, slit-lamp biomicroscopy, intraocular pressure, dilated fundoscopic examination was conducted.

EDI mode of spectral-domain OCT (SD-OCT) (Spectralis; Heidelberg Engineering, Heidelberg, Germany) images were performed one month after PPV with SO injection and two months $(8 \pm 2$ weeks) after SO removal. The choroidal thicknesses were measured by two independent graders manually. The average measurements were used in statistical analysis. Considering the diurnal variation in choroidal thickness, all SD-OCT measurements were performed between 09:00 and I 1:00 am. The choroid was measured from the hyper-reflective line corresponding to the retinal pigment epitheliumBruch's membrane complex to the sclera/choroidal junction. EDI mode SD-OCT images which were performed one month following PPV and SO injection and two months following SO removal surgery due to macula-off RRD are seen (Fig. I).

Patients with ocular diseases, such as uveitis, glaucoma, high myopia, diabetic retinopathy and retinal vascular diseases and ocular surgery except for uncomplicated cataract surgery, which may affect choroidal thickness measurements, were excluded from this study. Twenty eyes of 20 patients were operated by the same surgeon. Internal limiting membrane peeling procedure was not performed to any patients. Three of seven phakic patients were treated with combined surgery (phacoemulsification cataract surgery and PPV with SO injection). 23-gauge Constellation PPV (Alcon Laboratories Inc., Fort Worth, TX, USA) and non-contact wide-angle viewing system (Resight ${ }^{\circledR}$, Carl Zeiss Meditec AG, Jena, Germany) were used in the surgery.

Operations were performed under local and general anesthesia. Phacoemulsification cataract surgery was conducted in cases that cataract would make surgery more difficult. After core vitrectomy, the vitreous base shaved with the aid of $360^{\circ}$ scleral indentation. Fluid-air exchange was performed, in necessary cases, perfluorocarbon was injected for reattachment of the retinal detachment, and subretinal fluid was drained from the retinal tear. Endolaser photocoagulation was applied around the retinal breaks and $360^{\circ}$ peripheral retina. Following air- $1000-\mathrm{cSt}$ SO exchange, sclerotomies and conjunctiva were sutured with $7-0$ and $8-0$ vicryl suture respectively at the end of the surgery.

Statistical analysis was performed using SPSS 21 software program. The variables were investigated using analytical methods (Kolmogorov-Smirnov and Shapiro-Wilk's tests) to determine whether or not they are normally distributed. Changes in choroidal thickness were evaluated using the Wilcoxon test. $\mathrm{P}<0.05$ was considered statistically significant.

\section{Results}

20 eyes of 20 patients ( 8 female, 12 male) were enrolled in this retrospective, observational study. The demographic data and BCVA values before PPV with SO injection and two months after SO removal are given in Table I. The mean age was $56.5 \pm 4.7$ (range: 50-67 years) years. 7 eyes were phakic, and 13 eyes were pseudophakic before PPV surgery. 3 of 7 phakic eyes were performed combined PPV with SO injection and phacoemulsification cataract surgery. BCVA increased after SO removal in all the patients.

Transient intraocular pressure elevation, which can be controlled by medical treatment was observed in three patients (15\%) following PPV operation. Fibrin reaction in the anterior chamber was determined after PPV in one patient, and it was controlled with topical steroid and mydriatic
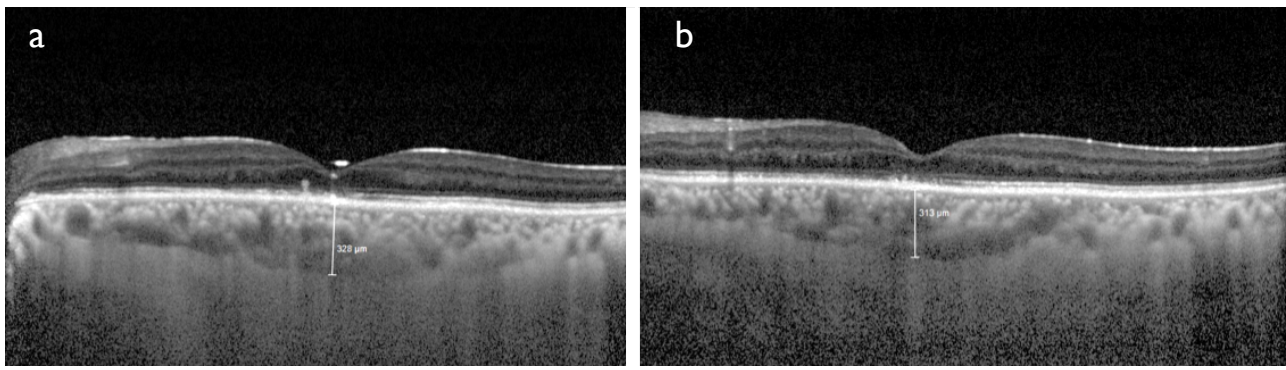

Figure I. Subfoveal choroidal thickness measurements with enhanced depth imaging (EDI) mode of spectral domain optical cohorence tomography (SD-OCT) which were taken one month after pars plana vitrectomy with silicone oil injection (a) and two months after silicone oil removal (b). 
Table I. Demographic data and best corrected visual acuity (Snellen) values of patients

\begin{tabular}{|c|c|c|c|c|c|}
\hline Patient & Age & Gender & Lens Status & Pre-PPV BCVA (Snellen) & $\begin{array}{l}\text { After Silicone Removal } \\
\text { BCVA (Snellen) }\end{array}$ \\
\hline I & 64 & M & $P$ & HM & 0.1 \\
\hline 2 & 60 & M & $P$ & HM & 0.2 \\
\hline 3 & 59 & M & $\mathrm{F}$ & 0.05 & 0.4 \\
\hline 4 & 60 & $\mathrm{~F}$ & $P$ & $\mathrm{IP+}$ & 0.05 \\
\hline 5 & 67 & $\mathrm{~F}$ & $\mathrm{~F}$ & HM & 0.3 \\
\hline 6 & 51 & M & $P$ & $50 \mathrm{cmCF}$ & 0.2 \\
\hline 7 & 59 & M & $P$ & HM & 0.5 \\
\hline 8 & 56 & M & $P$ & 0.05 & 0.6 \\
\hline 9 & 61 & M & $P$ & HM & 0.1 \\
\hline 10 & 54 & $\mathrm{~F}$ & $\mathrm{~F}$ & HM & 0.2 \\
\hline 11 & 52 & M & $P$ & $50 \mathrm{cmCF}$ & 0.4 \\
\hline 12 & 58 & $\mathrm{~F}$ & $P$ & $\mathrm{IP+}$ & 0.05 \\
\hline 13 & 52 & M & $P$ & $\mathrm{HM}$ & 0.2 \\
\hline 14 & 50 & $\mathrm{~F}$ & $\mathrm{~F}$ & $\mathrm{HM}$ & 0.3 \\
\hline 15 & 58 & $M$ & $\mathrm{~F}$ & $\mathrm{HM}$ & 0.5 \\
\hline 16 & 60 & $\mathrm{~F}$ & $P$ & 0.05 & 0.7 \\
\hline 17 & 55 & $M$ & $P$ & HM & 0.2 \\
\hline 18 & 53 & $\mathrm{~F}$ & $\mathrm{~F}$ & $\mathrm{HM}$ & 0.2 \\
\hline 19 & 52 & $M$ & $F$ & $50 \mathrm{cmCF}$ & 0.8 \\
\hline 20 & 50 & $\mathrm{~F}$ & $P$ & $\mathrm{IP}+$ & 0.1 \\
\hline
\end{tabular}

M: male; F: Female; P: Pseudophakic; F: Phakic; HM: Hand motionts; I+: light perception+; Pre-PPV BCVA: best corrected visual acuity before pars plana vitrectomy.

drops. None of the patients had hyphema, vitreous hemorrhage and endophthalmitis following PPV with SO injection and SO removal. Retinal layers were seen reattached at the end of the following period in all the patients.

The mean duration of the SO after PPV surgery was $109.6 \pm 19.9$ (range: 90 - 165 days) days. The mean subfoveal choroidal thickness was $284.2 \pm 54.2 \mu \mathrm{m}$ at one month after PPV with SO injection and $258.8 \pm 52.8 \mu \mathrm{m}$ at two months after SO removal. Subfoveal choroidal thickness changes were statistically significant ( $p<0.00$ I, Wilcoxon).

\section{Discussion}

In this retrospective observational study, we observed a decrease in subfoveal choroidal thickness in the second month ( $8 \pm 2$ weeks) following SO removal compared to the first month after the PPV and SO injection in patients who underwent PPV and SO injection due to RRD. Several studies have shown that choroidal thickness decreases after PPV (7-9). In a study regarding the changes in choroidal thickness in patients undergoing PPV and C3F8 injection due to RRD with macular involvement, it has shown that the subfoveal choroidal thickness has not changed (I0). This report supports that the decrease in subfoveal choroidal thickness in SO injected eyes is not related to PPV procedure, and it may be related to SO.

Due to the retrospective design of our study, preoperative subfoveal choroidal thickness measurements of the patients could not be obtained. However, Sugawara et al. (II) showed no significant change in subfoveal choroidal blood flow in a study of patients with macula-off RRD, which suggests that the decrease in subfoveal choroidal thickness is not associated with the disease.

Decreased choroidal thickness in patients with $\mathrm{SO}$ can be related to ischemia in choroid due to mechanical pressure of SO. Some authors argue that SO toxicity may arise from the failure of potassium siphoning by Muller cells and replacement of vitreous cavity with SO might deteriorate normal ion exchange between the retina and vitreous. Increasing intraretinal potassium causes thinning of inner retinal layers and degeneration of outer retinal layers. Alteration of this ion and fluid flow between the retina, RPE, and choroid may affect the choroidal thickness (I2). 
In a study conducted in enucleated siliconized eyes, the presence of macrophages, giant cells with lipid vacuoles suggest that choroid may be affected secondary to inflammation (4). We presume that long-time inflammation, such as eyes with emulsified silicone, may be responsible for choroidal thinning. SO may contribute to thinning of the retina and choroid with direct toxic effect.

The choroid provides oxygen and nutrients to the outer retinal layers (13). Because the foveolar avascular zone has no vasculature, it receives nutrients and oxygen from the choroidal circulation (I3). A stable temperature of outer retinal layers can be maintained with the aid of choroid (14). Thinning in choroid may affect all these functions and the visual prognosis of the patients. Therefore, we think that SO removal timing may be important for final visual acuity.

Karimi et al. (7) observed decreasing subfoveal choroidal thickness only after six months following PPV and SO injection for pseudophakic RRD, while Odrobina et al. indicated decreasing subfoveal choroidal thickness after three months following PPV and SO injection for retinal detachment with proliferative vitreoretinopathy. They emphasized that choroidal thinning at three months might be related to the surgical procedure. Since the incidence of retinectomy was higher in patients with proliferative vitreoretinopathy, they stressed that retinectomy might cause this result by causing deterioration in choroidal circulation. In our study, the mean duration of SO was 109.6 19.9 days, and we showed that subfoveal choroidal thickness decreased after removal of SO.

To our knowledge, there is not any study regarding the reliability of choroidal thickness measurements in siliconized eyes. A study reported that there were no statistically significant differences in foveal thickness measurements before and after SO removal; thus, the presence of SO in the vitreous chamber did not affect the reliability and reproducibility of foveal thickness measurements by OCT (15). Further prospective studies are needed for the reliability of choroidal thickness measurements in siliconized eyes.

The retrospective design of our study, the relatively low number of cases and the short follow-up period are limitations of our study. The lack of preoperative choroidal thickness measurements of patients due to retrospective design is also a factor that prevents further detailed comparisons.

In conclusion, monitoring of changes in subfoveal choroidal thickness may be a valuable indicator for the timing of SO removal. In light of all this information, it can be a rational approach to remove $\mathrm{SO}$ as soon as possible after showing reattachment of retinal layers with OCT in RRD patients who underwent PPV and SO injection.

\section{Disclosures}

Ethics Committee Approval: University of Health Sciences, Okmeydani Training and Research Hospital, March 19, 2019, no: II87.

Peer-review: Externally peer-reviewed.

Conflict of Interest: None declared.

Authorship Contributions: Involved in design and conduct of the study (SB); preparation and review of the study (SB, BE,AC); data collection (SB, BE, AC); and statistical analysis (SB).

\section{References}

I. Foulks GN, Hatchell DL, Proia AD, Klintworth GK. Histopathology of silicone oil keratopathy in humans. Cornea 1991;10:2937. [CrossRef]

2. Honavar SG, Goyal M, Majji AB, Sen PK, Naduvilath T, Dandona L. Glaucoma after pars plana vitrectomy and silicone oil injection for complicated retinal detachments. Ophthalmology 1999; 106: 169-76. [CrossRef]

3. Budde M, Cursiefen C, Holbach LM, Naumann GO. Silicone oil-associated optic nerve degeneration. Am J Ophthalmol 2001;|31:392-4. [CrossRef]

4. Wickham L, Asaria RH, Alexander R, Luthert P, Charteris DG. Immunopathology of intraocular silicone oil: enucleated eyes. Br J Ophthalmol 2007;91:253-7. [CrossRef]

5. Christensen UC, la Cour M. Visual loss after use of intraocular silicone oil associated with thinning of inner retinal layers. Acta Ophthalmol 2012;90:733-7. [CrossRef]

6. Budde M, Cursiefen C, Holbach LM, Naumann GO. Silicone oil-associated optic nerve degeneration. Am J Ophthalmol 2001;|31:392-4. [CrossRef]

7. Karimi S, Entezari M, Nikkhah H, Esfandiari H, Darvishpoor T, Tavakoli M, et al. Effects of Intravitreal Silicone Oil on Subfoveal Choroidal Thickness. Ophthalmologica 2018;239:159_ 66. [CrossRef]

8. Mirza E, Şatırtav G, Oltulu R, Kerimoğlu H, Gündüz MK. Subfoveal choroidal thickness change following pars plana vitrectomy with silicone oil endotamponade for rhegmatogenous retinal detachment. Int Ophthalmol 2018 Jul 24. [Epub ahead of print], doi: 10.1007/s 10792-018-0993-0. [CrossRef]

9. Odrobina D, Gołębiewska J, Maroszyńska I. Choroidal Thickness Changes After Vitrectomy With Silicone Oil Tamponade For Proliferative Vitreoretinopathy Retinal Detachment. Retina 2017;37:2124-9. [CrossRef]

10. Akkoyun I, Yilmaz G. Choroidal thickness after scleral buckling surgery versus pars plana vitrectomy in macula-off rhegmatogenous retinal detachment. [Article in German]. Klin Monbl Augenheilkd 2014;231:1029-33.

I I. Sugawara R, Nagaoka T, Kitaya N, Fujio N, Takahashi J, Takahashi $A$, et al. Choroidal blood flow in the foveal region in eyes with rhegmatogenous retinal detachment and scleral buckling procedures. Br J Ophthalmol 2006;90:1363-5. [CrossRef]

I2. Winter M, Eberhardt W, Scholz C, Reichenbach A. Failure of 
potassium siphoning by Müller cells: a new hypothesis of perfluorocarbon liquid-induced retinopathy. Invest Ophthalmol Vis Sci 2000;4I:256-6I.

13. Nickla DL, Wallman J. The multifunctional choroid. Prog Retin Eye Res 2010;29:144-68. [CrossRef]

14. Parver LM, Auker CR, Carpenter DO. The stabilizing effect of the choroidal circulation on the temperature environment of the macula. Retina 1982;2: I 17-20. [CrossRef]

15. Avitabile T, Bonfiglio V, Sanfilippo M, Torrisi B, Reibaldi A. Correlation of optical coherence tomography pattern and visual recovery after vitrectomy with silicone oil for retinal detachment. Retina 2006;26:917-2I. [CrossRef] 